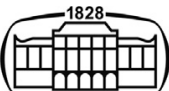

AKADÉMIAI KIADÓ

\title{
Potentially addictive behaviours increase during the first six months of the Covid-19 pandemic
}

\section{Journal of Behavioral Addictions}

10 (2021) 4, 912-919

D01:

$10.1556 / 2006.2021 .00079$

(c) 2021 The Author(s)

\author{
ANIKO MARAZ $^{1 *}$ (1), EVA KATZINGER ${ }^{1}$ (1) and \\ SUNGHWAN YI ${ }^{2} \odot$ \\ ${ }^{1}$ Institut für Psychologie, Humboldt-Universität zu Berlin, Germany \\ ${ }^{2}$ University of Guelph, Canada
}

Received: July 21, 2021 • Revised manuscript received: September 18, 2021; 0ctober 13, 2021 • Accepted: 0ctober 18, 2021

Published online: December 14, 2021

\section{FULL-LENGTH REPORT}

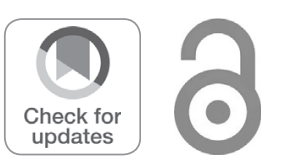

*Corresponding author. E-mail: aniko.maraz@gmail.com

\section{KEYWORDS}

chronic distress, addiction, alcohol use disorder, substance use disorder, pandemic, SARS-CoV-2

\section{INTRODUCTION}

The SARS-CoV-2 (Covid-19) pandemic and the measures taken to stop the spread of the virus has led to an unprecedented societal distress that affects billions, who experience fear, anxiety and social isolation over a prolonged period of time. Chronic stress is an established risk factor for the development of substance abuse disorders (Brady \& Sinha, 2005; Hedtke et al., 2008; Turner, Lloyd, \& Taylor, 2006) as well as for non-substance use disorders (Coman, Burrows, \& Evans, 1997). This finding is confirmed by several neuropsychological evidence. For example, chronic stress causes the basolateral amygdala output neurons to become hyperexcitable, which results in the altering of natural reward perception and to drug-conditioned cues (Sharp, 2017). Isolation, or reduced-stimulus environment combined with single prolonged stress increases self-administration of cocaine-intake in rats, which was more rapid than in a stressful, but non-isolated environment (Hofford, Prendergast, \& Bardo, 2018). To correspond with the current trend in the literature we adopted the term "potentially addictive behaviours" to reflect the shared etiology and potential overlap between substance and non-substance use disorders (Kotyuk et al., 2020).

There is evidence that people seek substitutes when a ban is introduced on relaxing, but potentially addictive activities such as alcohol and cigarette consumption in South Africa 
(Sinclair et al., 2021) and similar dynamics might take place during the current pandemic. Supporting this assumption, there are reports that the frequency of engaging in activities that are typically engaged in in order to relieve stress increased during lockdowns. For example, porn sites (Mestre-Bach, Blycker, \& Potenza, 2020), and online gaming initiatives (King, Delfabbro, Billieux, \& Potenza, 2020) reported an unprecedented increase of usage over the world. Furthermore, compared to before the Covid-19 outbreak, schoolchildren spent significantly more time on their smartphones (about $1 \mathrm{~h}$ more time a day) and on social media (Chen et al., 2021). Online retail also rocketed due to the pandemic: the largest online retail company, Amazon increased it revenue (net sales) by $29 \%$ in 2020 Q2 and by $27 \%$ in Q3 (from \$63.4 in 2019 Q2 to \$88.9 in 2020 Q2 ${ }^{1}$ and from $\$ 70.0$ billion in 2019 to $\$ 96.1$ in $2020^{2}$ ). About $8.2 \%$ in Q2 was probably due to the pandemic alone (expected $\$ 81.6$, but realised $\$ 88.9$ billion $\left.^{1}\right)$. According to a recent metaanalysis, smoking also increased with the progression of Covid-19 especially among those who were already smokers before the outbreak (Patanavanich \& Glantz, 2020). Over half of participants reported snacking more frequently and struggling with keeping a diet compared to before the lockdown in the UK (Robinson et al., 2021) and almost half of an Italian sample gained weight during the lockdown (Di Renzo et al., 2020). Similarly alarming rates were observed in gambling (Håkansson, Fernández-Aranda, Menchón, Potenza, \& Jiménez-Murcia, 2020). Besides contributing to well-known health problems, heavy alcohol consumption also reduces immunity to viral and bacterial infections (Szabo \& Saha, 2015), and yet Polish drinkers increased their consumption after the Pandemic started, especially those with less adaptive coping strategies (Chodkiewicz, Talarowska, Miniszewska, Nawrocka, \& Bilinski, 2020). Substance use was also found to increase after the beginning of the pandemic, especially among those with pre-Covid-19 substance users, and this increase was found to be related to the intensity of worry they held about Covid situations (Rogers, Shepherd, Garey, \& Zvolensky, 2020). In a representative sample administered in late June 2020 in the US, authors reported that $13.3 \%$ of respondents increased their substance use in an attempt to cope with Covid-19 related stress (Czeisler et al., 2020).

There is evidence that suggests that addiction is an attempt to adapt to interpersonal trauma, as a result of "compromised abilities to form healthy attachments and decreased capacity for self-regulation” (p. 352. Padykula \& Conklin, 2010). For example, alcohol use may increase the distress as a result of endorphin withdrawal following exposure to trauma and maintain alcohol abuse (Volpicelli, Balaraman, Hahn, \& Bux, 1999). Furthermore, symptoms of PTSD are associated with an increased prevalence of food

\footnotetext{
${ }^{2}$ https://s2.q4cdn.com/299287126/files/doc_financials/2020/q3/AMZN-Q3-2020Earnings-Release.pdf.

${ }^{1}$ https://s2.q4cdn.com/299287126/files/doc_financials/2020/q2/Q2-2020Amazon-Earnings-Release.pdf.
}

addiction in women (Mason et al., 2014) and gaming disorder symptoms (Kircaburun, Griffiths, \& Billieux, 2019) among others.

The pandemic and the subsequent lockdowns had an unprecedented effect on people's quality of life. The prolonged fear of the disease, unavailability of other, healthier coping strategies (e.g., socialising, physical exercise with others), as well as restrictions on recreational traveling, and serious reduction in social interactions all impacted wellbeing. This has resulted in increased and chronic distress, especially for those with a pre-existing mental health condition. Addictive or potentially addictive behaviours are likely to be a mental and physical attempt to escape the situation at hand as a way of coping with distress (Király, Tóth, Urbán, Demetrovics, \& Maraz, 2017).

The current Covid-19 situation offers a natural experiment to behavioural addiction researchers, and therefore provides a unique opportunity to gain insight into the effects of chronic stress on potentially addictive behaviours. There is strong evidence, for example, that depressive and anxiety symptoms increased before (T1) to during the pandemic (T2) in children and adolescents, and this positively predicted internet gaming disorder (Teng, Pontes, Nie, Griffiths, \& Guo, 2021). This effect was mediated by the perceived Covid-19 impact on different life domains (i.e., study activities, sleep quality, lifestyle habits), although the strongest predictor of gaming activity at T2 was gaming reported at T1. Therefore, the question remains as to how excessive behaviours change over the course of Covid-19 pandemic, and whether these changes are related to the rise of daily distress experienced by adults during this period of time. To our knowledge, this is the first study that assessed multiple potentially addictive behavioural problems simultaneously and over an extended period of time to allow relative and quasi-longitudinal assessment of trends in this domain.

Our hypotheses for the study were the following: (H1) The frequency of self-reported potentially addictive behavioural problems would increase over time as distress becomes chronic. (H2) The frequency of self-reported potentially addictive behavioural problems would be related to the intensity of distress experienced by individuals. As an exploratory aim we will study the strength of relationship between the potentially addictive behavioural problems and distress as the period of lockdown progresses.

\section{METHODS}

\section{Participants and procedure}

Data were collected every three days from Amazon's MTurk between March 26, 2020 and October 2, 2020 (inclusive) covering a period of 191 days (from Day 15 till Day 206 of the pandemic as declared on the 11th March 2020 by the WHO). Each time a new sample of 25 participants was recruited excluding the participants who had previously taken part. Participants were able to take part if they were above the age of 18 (as verified by Amazon) and they were logged in from a US-based IP address. 


\section{Measures}

Potentially addictive behavioural problems were assessed with a single item: "How often did you engage in [i.e. gaming] in the past seven days? Five alternatives were offered: "too much", "quite a lot", "somewhat", "a little bit", "not at all". Pre-designed behaviours were the following: shopping, alcohol, smoking, legal substance(s), illegal substance(s) (optional), gambling, gaming, overeating. All but shopping was assessed from day 15 of the outbreak. Shopping was assessed on day 41 onwards. A ninth, "other" category was also offered so that participants could name an activity and report the frequency of engaging in the behaviour.

Distress was addressed via the 14-item Perceived Stress Scale (Cohen, Kamarck, \& Mermelstein, 1983). This instrument defaults to the past month, but was modified to the past seven days in the current survey. The PSS asks how often the person experienced the listed feelings from "Never" $(=1)$ to "Very often" (=5). McDonald's omega of factor saturation was 0.84 (Dunn, Baguley, \& Brunsden, 2014).

Covid-19 related stress was assessed with a single item: "How stressful do you feel about the current situation caused by the corona virus outbreak?" Rating was given on a scale of 1 (Not at all stressful) to 10 (Very stressful). Correlation between the PSS and the Covid-19 related distress was $\tau=0.31(P<0.001)$ (Kendall's rank correlation).

\section{Attention check items and statistical analysis}

Three attention check items were hidden among regular items (e.g. "Please check “true" here."). Participants' age was asked twice, and they scored an error if the answers were different. Those scoring more than one (out of 4) attentional error were excluded from the sample. Finally, those with maximal score (10 out of 10) on the Marlowe-Crowne Social Desirability Scale (Strahan \& Gerbasi, 1972) were also excluded. By design all non-sensitive survey items were obligatory.

Overall 1885 people started filling out the questionnaire and 1,605 finished with no missing data. After excluding those with more than one error (145) and those with 10/10 lie score (30), 1,430 participants' data were left for the analyses.

Kendall's tau $(\tau)$ was used in order to test the associations between an ordered and a continuous, or two ordered variables (i.e. the day of assessment and 5-point scale of frequency of the given behavioural addiction), which can handle ties in the data where members of the pair have the same ordinal value.

Data were collected using formr (Arslan, Walther, \& Tata, in press). We analysed and visualised data in $R$ (R Core Team, 2013) using the psych (Revelle, 2020) and the ggplot2 packages (Wickham, 2016). Smoothing was used for trend visualising, using the locally estimated scatterplot smoothing (LOESS) method with span $=0.2$ to reduce noise.

\section{Ethics and preregistration}

The study procedures were carried out in accordance with the Declaration of Helsinki. All participants were informed about the study in compliance with the GDPR, and all provided informed consent. Ethical permission was granted by the ERB prior to data collection (2020-15R3).

The data collection procedure for the study was preregistered prior to data collection (https://osf.io/m5kw9). This paper uses part of the data collected for the study. All data and scripts of analyses are available open access on the OSF: https://osf.io/qdhp4/ and under https:/github.com/ anikomaraz/shopping_covid 19 .

\section{RESULTS}

Overall, 1,430 participants' data were left for the analyses. There were 562 women (39\%) and 858 (60\%) men in the sample (seven participants did not identify with any gender and three did not want to answer). Mean age was 36.6 (SD $=11$ ) years. In terms of education most people had graduate education ( $n=615), 359$ had higher and the rest had lower educational Overall, participants reported slightly living below the mean standard of living (see Fig. 2 from https:// www.researchsquare.com/article/rs-640061).

As seen in Fig. 1, in the total sample (disregarding time) most participants reported "other" potentially addictive behaviours as the most disturbing. For the other category, they reported a range of stress-related behaviours from probably benign ones (e.g., "speaking too much", "singing") to possibly potentially addictive ones (e.g., "over-exercising", "Wasting my working time, not getting enough accomplished"). Other examples included "masturbation", "too much web browsing", "playing with my kids", "lying", the relevance and severity of which was difficult to assess (see the data file for the full list of behaviours). The second most common self-identified problematic behaviours were gaming and shopping, followed by overeating, smoking, legal substance use, gambling, illegal substance use and finally alcohol use ${ }^{3}$.

Following fluctuations at the initial of the pandemic outbreak, distress reported by our participants steadily increased from about Day 80 when the number of Covid-19 cases started to increase (see Fig. 2). The pattern of increase stopped at around Day 140 as the first wave of pandemic reached its peak. However, although distress started to go down afterwards, it did not dissipate as quickly as the number of confirmed covid cases decreased. The single-item measure of Covid-19 related distress and the multi-item measure of general distress (PSS) for the same period appeared to follow the same pattern suggesting that the distress for this period was closely related to Covid-19 stress. Correlation between the PSS and the Covid-19 related distress in the total sample was $\tau=0.31(P<0.001)$.

$\mathrm{H} 1$ concerned the frequency of potentially addictive behavioural problems over time. As depicted in Fig. 2., the

${ }^{3}$ Testing the overlap between potentially addictive behaviours would have too many ties, thus it would have resulted in under-powered statistical outcomes. 


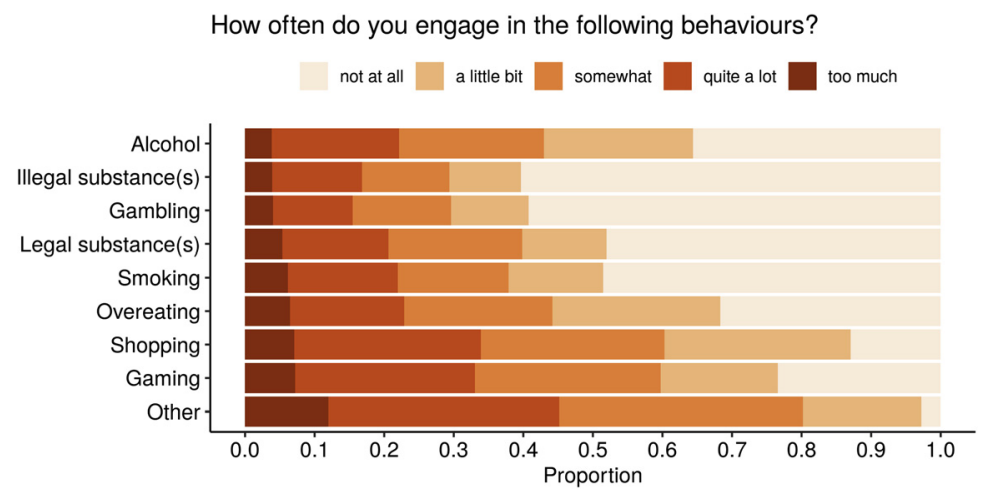

Fig. 1. Frequency of the reported potentially addictive behaviours during the data collection period

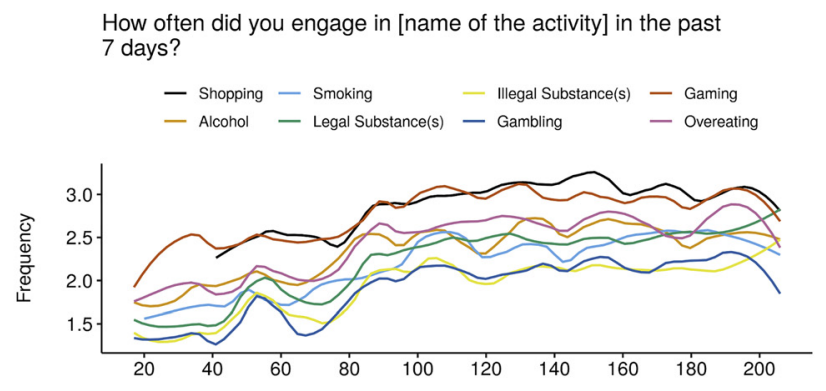

Distress during the outbreak
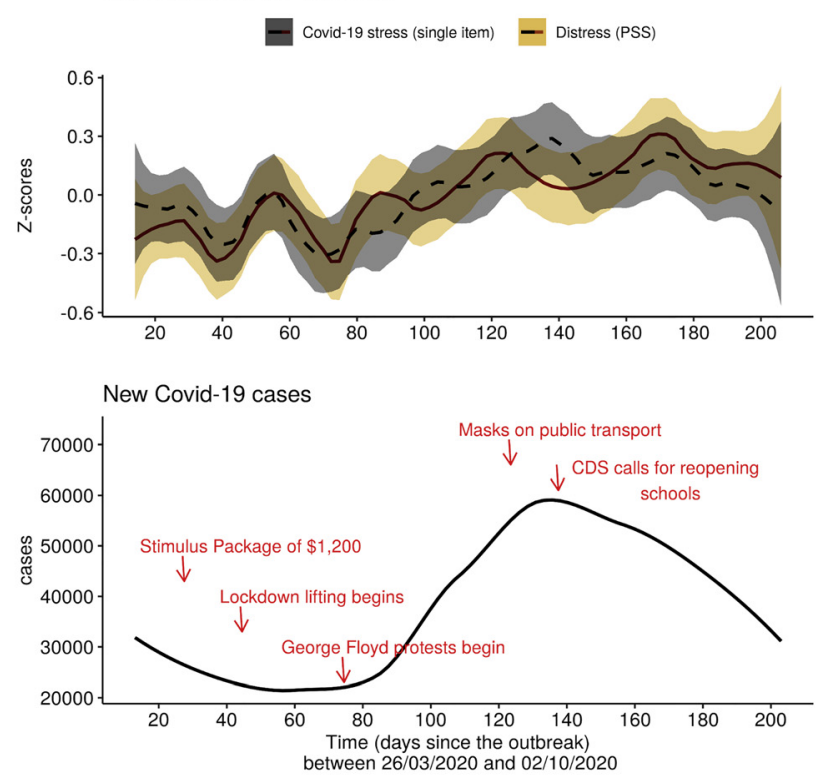

Fig. 2. Potentially addictive behaviours during the outbreak

frequency of each self-identified problematic behaviour increased over time. This was particularly the case following the steep rise in case numbers in the US in early July when both distress and Covid-19 related stress increased along with the reported potentially addictive behaviours. In terms of the statistics, there is a linear relationship between time, and potentially addictive shopping $(\tau=0.15, P<0.001)$, alcohol $(\tau=0.17, P<0.001)$, smoking $(\tau=0.18, P<0.001)$, legal drug (medicine) use $(\tau=0.23, P<0.001)$, illegal drug use $(\tau=0.19, P<0.001)$, gambling $(\tau=0.22, P<0.001)$, gaming $(\tau=0.14, P<0.001)$ and overeating $(\tau=0.19, P<$ $0.001)$ in the total sample.

The second hypothesis regarded the relationship between self-reported potentially addictive behavioural problems and distress. Kendall's correlation indicated a linear relationship between distress and the frequency of each potentially addictive behaviour: shopping $(\tau=0.20, P<0.001)$, alcohol ( $\tau=0.22, P<0.001)$, smoking $(\tau=0.18, P<0.001)$, legal drug (medicine) use $(\tau=0.25, P<0.001)$, illegal drug use ( $\tau=0.23, P<0.001)$, gambling $(\tau=0.25, P<0.001)$, gaming $(\tau=0.12, P<0.001)$ and overeating $(\tau=0.28, P<0.001)$ in the total sample. Fig. 3 demonstrated the correlations at each point of time between distress (as measured by the PSS) and the frequency of the self-reported potentially addictive behaviour. As a trend, the correlation with distress decreases within shopping and eating, remained about the same in potentially addictive alcohol use, gambling and gaming, whereas in the remaining behaviours the trend was less clear.

Similarly to the multi-item distress measure, Kendall's rank correlation also detected significant relationship between the one-item Covid-19 related distress and the listed potentially addictive behaviours: shopping $(\tau=0.27, P<$ $0.001)$, alcohol $(\tau=0.22, P<0.001)$, smoking $(\tau=0.19, P<$ $0.001)$, legal drug (medicine) use $(\tau=0.25, P<0.001)$, illegal drug use $(\tau=0.23, P<0.001)$, gambling $(\tau=0.23, P<$ $0.001)$, gaming $(\tau=0.16, P<0.001)$ and overeating $(\tau=0.28, P<0.001)$ in the total sample.

\section{DISCUSSION}

We found that the frequency of self-reported potentially addictive behaviours (shopping, alcohol use, smoking, legal and illegal drug use, gambling, gaming and overeating) significantly increased over time during the first six months of the Covid-19 outbreak in a sample of 1,430 US adults. Furthermore, self-reported potentially addictive behavioural problems are linearly related to the intensity of distress, both general and Covid-19 related, especially in legal drug use, gambling and over-eating. Most popular activities during the data collection period were gaming and shopping excessively, although other potentially addictive behaviours also increased in their frequency. The popularity of each 


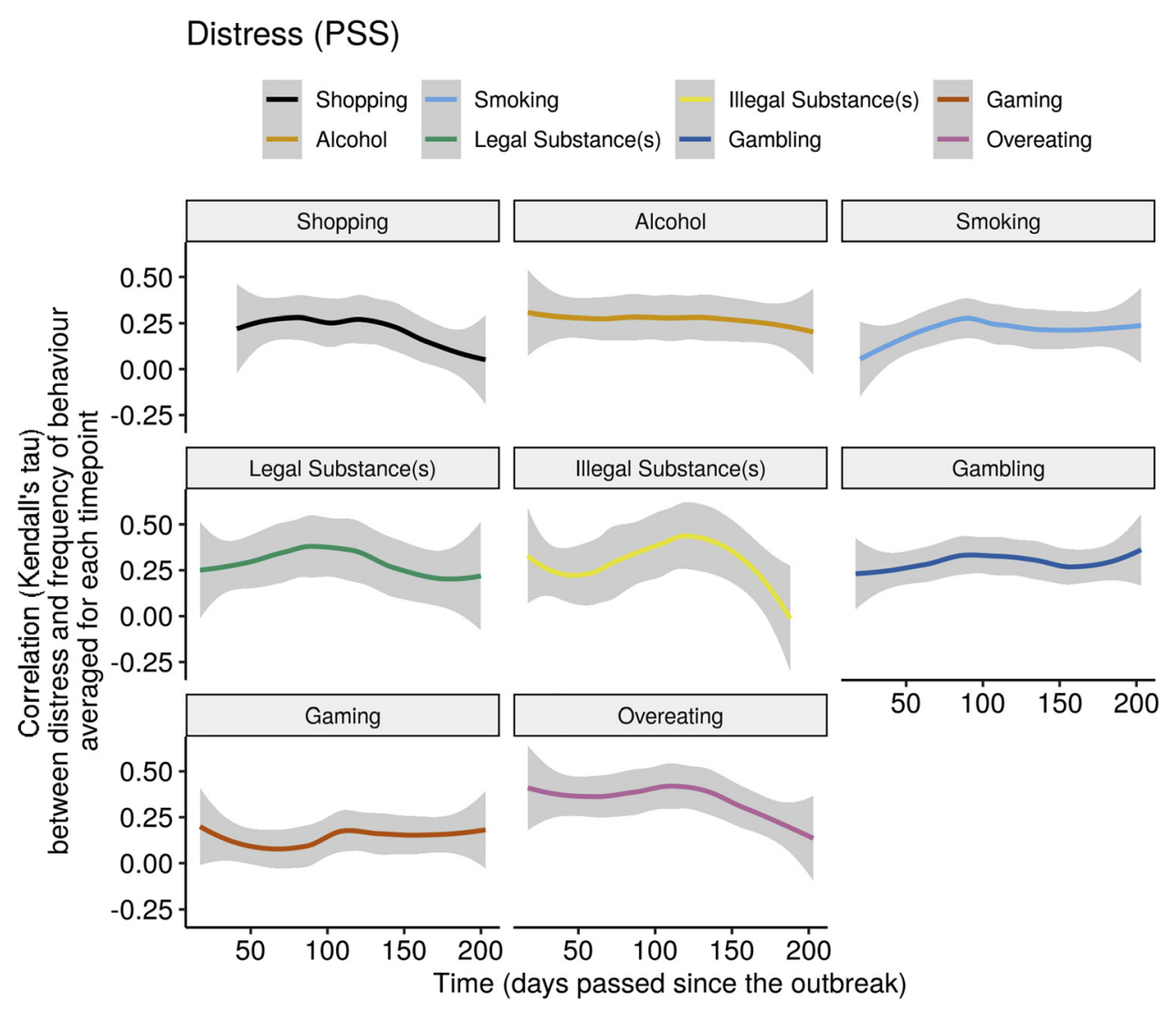

Fig. 3. The strength of correlation over time between distress and each potentially addictive behaviour

potentially addictive behaviour remained the same relative to each other during the data collection period.

Although self-reported potentially addictive use of a substance or over-indulging in a behaviour cannot be considered as an addiction per se, it may serve as a proxy to truly problematic behaviour. Thus, it helps increase our understanding of the effect of prolonged distress both related and unrelated with the pandemic. Throughout the data collection period the two most self-reported potentially addictived behaviours were shopping and gaming, although all measured behaviours increased during the first six months of the pandemic including alcohol use, smoking, legal and illegal substance use, as well as gambling and overeating. Distress gradually increased from around Day 70 when the case numbers started to increase, and the reported frequency of excessive behaviours also followed this increase. Finally, there is a stagnating, then declining trend in the potentially addictive behaviours beyond about 150 days, which might be due to the decreased intensity of distress and Covid-related stress due to the decreasing number of new Covid-19 cases.

Our findings are in line with other studies, which used extensive (multi-item) measures and also found an increase in gaming (King et al., 2020), compulsive buying (King et al., 2020), smoking (Patanavanich \& Glantz, 2020), overeating (Robinson et al., 2021), gambling (Håkansson et al., 2020), drinking alcohol (Chodkiewicz et al., 2020), and in substance use (Czeisler et al., 2020; Rogers et al., 2020), although most studies used a retrospective self-reported comparison or a short period of time to conclude the increasement in the given behaviour. Our data shows that the increase of the potentially addictive behaviours happened gradually but constantly over the measured period, and closely followed the Covid-19 related distress and increase in new cases. Furthermore, our data showed that the most problematic behaviours relative to the others are gaming and shopping, which topped the list of potentially addictive behaviours throughout the data collection period. Therefore, these behaviours have to be studied carefully during and after the pandemic especially in those who were prone to abuse of gaming or of shopping before the pandemic. Perhaps the reason for the increasing potentially addictive behaviours during the pandemic is that people seek substitutes when other coping mechanisms are blocked, such as social recreation or most forms of exercise (Sinclair et al., 2021). Although the current study ended at day 191 of the pandemic, it is possible that frequency of engaging in addictive behaviours may increase again with another wave of Covid-19 crisis accompanying lockdown or other public measures intended to reduce social gatherings and/or outdoor activities.

Additionally, our data revealed a direct, linear relationship between distress (both general and Covid-19-related) and potentially addictive behaviours. This is also in line with previous studies in the field (Chodkiewicz et al., 2020; Czeisler et al., 2020; Rogers et al., 2020; Teng et al., 2021). 
Distress was a strong predictor of compulsive buying, alcohol use, legal and illegal substance use, gambling and overeating, while gaming and smoking appeared to be the least related to distress on both types of stress measures. Based on the data, it appears that in the first few months of the pandemic, the relationship between the potentially addictive behaviour and distress appeared to be stronger, than at later stages of the outbreak. This is in line with the theory that suggests that addictive behaviours are an attempt to adapt to interpersonal trauma (Padykula \& Conklin, 2010), especially because most measures were introduced to reduce personal contact with the aim of slowing the spread of the pandemic.

As an exploratory study we calculated the correlations between distress and the frequency of each potentially addictive behaviour. For alcohol, gambling, gaming and to a lesser extent smoking the trend remained about constant, whereas the correlation between distress and compulsive buying, overeating, legal and illegal substance use appeared to be stronger during the first few months of the pandemic compared to the last few months of the data collection period. Therefore it is possible that excessive behaviours used to cope with distress change during the pandemic, or the effect of other factors (changes in employment, close relationships or as a result of governmental measures) modify coping opportunities or motivation of people. Addiction as an adaptation theory would suggest (Padykula \& Conklin, 2010), that following the first months of elevated coping attempts, self-control may fatigue some individuals, while others are better able to mostly be in control over their habits, which may explain the decreasing strength of correlation for some behaviours in our sample.

The main limitation of this study is the one-item selfreported assessment of the potentially addictive behaviour in question. Although this reporting is prone to individual differences in behavioural self-perception, it may serve as a proxy for truly problematic behaviour without burdening participants with multiple detailed questionnaires. Related to this is the limitation that the current design was unable to distinguish truly problematic from self-reported problematic behaviour. A further constraint is the lack of follow-up design, i.e. that a different sample of people was surveyed every time. We decided on this approach in order to avoid fatigue, dropout and the impact of individual effects (i.e. becoming unemployed) in the sample, however, a new sample is not a longitudinal assessment of prolonged effects. Furthermore, one should only cautiously generalise our findings to non-MTurkers, although there is evidence, that after careful attention check and data cleaning measures, MTurk samples are more informative about the general population than most ad-hoc gathered (especially panel) samples (Kennedy et al., 2020; Smith, Roster, Golden, \& Albaum, 2016). Finally, despite the significant correlations, it is possible, that third factors might mediate the effect of distress, such as health and economic fears (Eger, Komárková, Egerová, \& Mičík, 2021), true problematic abuse of the behaviour (Chen et al., 2021), the de-facto cohabiting status (i.e. living alone or with family) the quality of social network (Lopes \& Jaspal, 2020), or pre-pandemic levels of use or abuse (Rogers et al., 2020).

In conclusion, the frequency of self-reported potentially addictive behaviours increased during the first six months of the pandemic (especially gaming and shopping relative to the other behaviours), and the increase is closely related to Covid-19 and general distress. Individuals, who are affected with elevated levels of problematic behaviour may experience decreased availability of both social and health care resources even after the pandemic resolves, especially those with a diagnosed mental health disorder, who display more fear of Covid-19 than those without a diagnosis (Jaspal, Lopes, \& Lopes, 2020). Therefore, it is important to screen for truly excessive, potentially addictive behaviours during the pandemic and special attention needs to be paid to reduce potential harmful effects of maladaptive coping during this demanding period and thereafter.

Funding sources: Institute of Psychology at the Humboldt University of Berlin. We further acknowledge support by the German Research Foundation (DFG) and the Open Access Publication Fund of Humboldt-Universität zu Berlin.

Authors' contribution: AM designed the concept and delivery of the study, wrote the statistical analysis and interpreted the data. EK helped with data analysis. YS provided consultation on the design and interpretation of findings. All authors had full access to all data in the study and they all take responsibility for the integrity of the data and the accuracy of the data analysis.

Conflict of interest: The authors declare no conflict of interest.

\section{REFERENCES}

Arslan, R. C., Walther, M., \& Tata, C. (in press). formr: A study framework allowing for automated feedback generation and complex longitudinal experience sampling studies using $R$. https://doi.org/10.31234/osf.io/pjasu.

Brady, K. T., \& Sinha, R. (2005). Co-occurring mental and substance use disorders: The neurobiological effects of chronic stress. American Journal of Psychiatry, 162(8), 1483-1493. https://doi.org/10.1176/appi.ajp.162.8.1483.

Chen, I.-H., Chen, C.-Y., Pakpour, A. H., Griffiths, M. D., Lin, C.-Y., Li, X.-D., \& Tsang, H. W. H. (2021). Problematic internet-related behaviors mediate the associations between levels of internet engagement and distress among schoolchildren during COVID-19 lockdown: A longitudinal structural equation modeling study. Journal of Behavioral Addictions. https://doi.org/10.1556/2006.2021.00006.

Chodkiewicz, J., Talarowska, M., Miniszewska, J., Nawrocka, N., \& Bilinski, P. (2020). Alcohol consumption reported during the COVID-19 pandemic: The initial stage. International Journal of 
Environmental Research and Public Health, 17(13), 4677. https://doi.org/10.3390/ijerph17134677.

Cohen, S., Kamarck, T., \& Mermelstein, R. (1983). A global measure of perceived stress. Journal of Health and Social Behavior, 24(4), 385. https://doi.org/10.2307/2136404.

Coman, G. J., Burrows, G. D., \& Evans, B. J. (1997). Stress and anxiety as factors in the onset of problem gambling: Implications for treatment. Stress Medicine, 13(4), 235-244.

Czeisler, M. É., Lane, R. I., Petrosky, E., Wiley, J. F., Christensen, A., Njai, R., . . Rajaratnam, S. M. W. (2020). Mental health, substance use, and suicidal ideation during the COVID-19 pandemic-United States, June 24-30, 2020. MMWR. Morbidity and Mortality Weekly Report, 69(32), 1049-1057. https://doi.org/10.15585/mmwr.mm6932a1.

Di Renzo, L., Gualtieri, P., Pivari, F., Soldati, L., Attinà, A., Cinelli, G., .. De Lorenzo, A. (2020). Eating habits and lifestyle changes during COVID-19 lockdown: An Italian survey. Journal of Translational Medicine, 18(1), 229. https://doi.org/10. 1186/s12967-020-02399-5.

Dunn, T. J., Baguley, T., \& Brunsden, V. (2014). From alpha to omega: A practical solution to the pervasive problem of internal consistency estimation. British Journal of Psychology, 105(3), 399-412. https://doi.org/10.1111/bjop.12046.

Eger, L., Komárková, L., Egerová, D., \& Mičík, M. (2021). The effect of COVID-19 on consumer shopping behaviour: Generational cohort perspective. Journal of Retailing and Consumer Services, 61, 102542. https://doi.org/10.1016/j.jretconser.2021.102542.

Håkansson, A., Fernández-Aranda, F., Menchón, J. M., Potenza, M. N., \& Jiménez-Murcia, S. (2020). Gambling during the COVID-19 crisis - a cause for concern. Journal of Addiction Medicine, 14(4), e10-e12. https://doi.org/10.1097/ADM.0000000000000690.

Hedtke, K. A., Ruggiero, K. J., Fitzgerald, M. M., Zinzow, H. M., Saunders, B. E., Resnick, H. S., \& Kilpatrick, D. G. (2008). A longitudinal investigation of interpersonal violence in relation to mental health and substance use. Journal of Consulting and Clinical Psychology, 76(4), 633-647. https://doi.org/10. 1037/0022-006X.76.4.633.

Hofford, R. S., Prendergast, M. A., \& Bardo, M. T. (2018). Modified single prolonged stress reduces cocaine self-administration during acquisition regardless of rearing environment. Behavioural Brain Research, 338, 143-152. https://doi.org/10.1016/j. bbr.2017.10.023.

Jaspal, R., Lopes, B., \& Lopes, P. (2020). Predicting social distancing and compulsive buying behaviours in response to COVID-19 in a United Kingdom sample. Cogent Psychology, 7(1), 1800924. https://doi.org/10.1080/23311908.2020.1800924.

Kennedy, R., Clifford, S., Burleigh, T., Waggoner, P. D., Jewell, R., \& Winter, N. J. G. (2020). The shape of and solutions to the MTurk quality crisis. Political Science Research and Methods, 8(4), 614-629. https://doi.org/10.1017/psrm.2020.6.

King, D. L., Delfabbro, P. H., Billieux, J., \& Potenza, M. N. (2020). Problematic online gaming and the COVID-19 pandemic. Journal of Behavioral Addictions, 9(2), 184-186. https://doi.org/ 10.1556/2006.2020.00016.

Király, O., Tóth, D., Urbán, R., Demetrovics, Z., \& Maraz, A. (2017). Intense video gaming is not essentially problematic. Psychology of Addictive Behaviors, 31(7), 807-817. https://doi. org/10.1037/adb0000316.
Kircaburun, K., Griffiths, M. D., \& Billieux, J. (2019). Psychosocial factors mediating the relationship between childhood emotional trauma and internet gaming disorder: A pilot study. European Journal of Psychotraumatology, 10(1), 1565031. https://doi.org/10.1080/20008198.2018.1565031.

Kotyuk, E., Magi, A., Eisinger, A., Király, O., Vereczkei, A., Barta, C., ... Demetrovics, Z. (2020). Co-occurrences of substance use and other potentially addictive behaviors: Epidemiological results from the Psychological and Genetic Factors of the Addictive Behaviors (PGA) Study. Journal of Behavioral Addictions, 9(2), 272-288. https://doi.org/10.1556/2006.2020.00033.

Lopes, B. C. da S., \& Jaspal, R. (2020). Understanding the mental health burden of COVID-19 in the United Kingdom. Psychological Trauma: Theory, Research, Practice, and Policy, 12(5), 465-467. https://doi.org/10.1037/tra0000632.

Mason, S. M., Flint, A. J., Roberts, A. L., Agnew-Blais, J., Koenen, K. C., \& Rich-Edwards, J. W. (2014). Posttraumatic stress disorder symptoms and food addiction in women by timing and type of trauma exposure. JAMA Psychiatry, 71(11), 1271. https://doi. org/10.1001/jamapsychiatry.2014.1208.

Mestre-Bach, G., Blycker, G. R., \& Potenza, M. N. (2020). Pornography use in the setting of the COVID-19 pandemic. Journal of Behavioral Addictions, 9(2), 181-183. https://doi.org/ 10.1556/2006.2020.00015.

Padykula, N. L., \& Conklin, P. (2010). The self regulation model of attachment trauma and addiction. Clinical Social Work Journal, 38(4), 351-360. https://doi.org/10.1007/s10615-009-0204-6.

Patanavanich, R., \& Glantz, S. A. (2020). Smoking is associated with COVID-19 progression: A meta-analysis. Nicotine \& Tobacco Research, 22(9), 1653-1656. https://doi.org/10.1093/ntr/ntaa082.

R Core Team. (2013). $R$ : A language and environment for statistical computing.

Revelle. (2020). Psych: Procedures for psychological, psychometric, and personality research (Version 2.0.9.).

Robinson, E., Boyland, E., Chisholm, A., Harrold, J., Maloney, N. G., Marty, L., ... Hardman, C. A. (2021). Obesity, eating behavior and physical activity during COVID-19 lockdown: A study of UK adults. Appetite, 156, 104853. https://doi.org/10. 1016/j.appet.2020.104853.

Rogers, A. H., Shepherd, J. M., Garey, L., \& Zvolensky, M. J. (2020). Psychological factors associated with substance use initiation during the COVID-19 pandemic. Psychiatry Research, 293, 113407. https://doi.org/10.1016/j.psychres.2020.113407.

Sharp, B. M. (2017). Basolateral amygdala and stress-induced hyperexcitability affect motivated behaviors and addiction. Translational Psychiatry, 7(8), e1194-e1194. https://doi.org/10. 1038/tp.2017.161.

Sinclair, D. L., Vanderplasschen, W., Savahl, S., Florence, M., Best, D., \& Sussman, S. (2021). Substitute addictions in the context of the COVID-19 pandemic. Journal of Behavioral Addictions, 9(4), 1098-1102. https://doi.org/10.1556/2006.2020.00091.

Smith, S. M., Roster, C. A., Golden, L. L., \& Albaum, G. S. (2016). A multi-group analysis of online survey respondent data quality: Comparing a regular USA consumer panel to MTurk samples. Journal of Business Research, 69(8), 3139-3148. https://doi.org/10.1016/j.jbusres.2015.12.002.

Strahan, R., \& Gerbasi, K. C. (1972). Short, homogeneous versions of the Marlow-Crowne social desirability scale, 28(2), 191-193. 
Szabo, G., \& Saha, B. (2015). Alcohol's effect on host defense. Alcohol Research: Current Reviews, 37(2), 159+.

Teng, Z., Pontes, H. M., Nie, Q., Griffiths, M. D., \& Guo, C. (2021). Depression and anxiety symptoms associated with internet gaming disorder before and during the COVID-19 pandemic: A longitudinal study. Journal of Behavioral Addictions. https:// doi.org/10.1556/2006.2021.00016.

Turner, R. J., Lloyd, D. A., \& Taylor, J. (2006). Physical disability and mental health: An epidemiology of psychiatric and substance disorders. Rehabilitation Psychology, 51(3), 214-223. https://doi.org/10.1037/0090-5550.51.3.214.

Volpicelli, J., Balaraman, G., Hahn, J., \& Bux, D. (1999). The role of uncontrollable trauma in the development of PTSD and alcohol addiction. Alcohol Research \& Health: The Journal of the National Institute on Alcohol Abuse and Alcoholism, 23(4), 256262.

Wickham, H. (2016). ggplot2: Elegant graphics for data analysis. Springer. Retrieved from http://ggplot2.org. 